\title{
Réflexions sur la proportion du lapin de garenne (Oryctolagus cuniculus) dans le régime alimentaire des chasseurs-cueilleurs autour du Dernier Maximum Glaciaire en Ibérie*
}

\author{
Rethinking the proportion of European rabbit (Oryctolagus cuniculus) in the diet of hunter- \\ gatherers around the Last Glacial Maximum in Iberia
}

Reflexiones sobre la proporción del conejo europeo (Oryctolagus cuniculus) en la dieta de
los cazadores-recolectores en torno al Último Máximo Glacial en Iberia

Samuel Seuru ${ }^{a}$, Ariane Burke ${ }^{a}$ et Liliana Perez

\begin{abstract}
RÉSUMÉ
Bien qu'aujourd'hui le lapin de garenne (Oryctolagus cuniculus) soit considéré comme une espèce invasive dans plusieurs pays, il fait l'objet d'un débat intense quant aux raisons du début de son exploitation durant le Paléolithique supérieur dans la péninsule ibérique. Cependant, aucun consensus général n'existe afin d'expliquer les causes de son introduction dans la diète des chasseurs-cueilleurs ibériens. Ici, nous présentons d'abord que sa chasse a débuté avant le Dernier Maximum Glaciaire (DMG) et qu'elle s'est accrue durant cette période. Ensuite, nous évaluons les principales hypothèses soutenues à partir du Modèle Classique du Régime Alimentaire (MCRA; Diet Breadth Model) issu de la Théorie de l'Approvisionnement Optimal (TAO; Optimal Foraging Theory). Bien que ce modèle soit à l'origine (parfois implicitement) de plusieurs de ces hypothèses, il n'a jamais été appliqué concrètement au DMG dans la péninsule. Nous avons alors appliqué ce modèle et les résultats obtenus ne semblent pas appuyer les hypothèses soutenant une intensification de l'exploitation de l'environnement. Plutôt, nous suggérons que l'utilisation de filets par plusieurs personnes pourrait expliquer l'exploitation de ce taxon autour du DMG en Ibérie.
\end{abstract}

\section{ABSTRACT}

Although today the European rabbit (Oryctolagus cuniculus) is considered invasive in several countries, reasons for the origins of its exploitation during the Upper Paleolithic in the Iberian peninsula are much debated. However, there exists no general consensus as to the causes of the introduction of the rabbit into the diet of hunter-gatherers in the past in Iberia. Here, we show that rabbit hunting started before the Last Glacial Maximum (LGM) and then increased during this period. We use the Diet Breadth Model from Optimal Foraging Theory to test current hypotheses. Although this model is at the origin (sometimes implicitly) of several of these hypotheses, it has never been concretely applied to the LGM in the peninsula. The results obtained in this research do not support hypotheses proposing an intensification of the exploitation of the environment. Rather, we suggest that the use of nets by several individuals is a valid hypothesis to explain the large number of European rabbit remains in archaeozoological sites around the LGM in Iberia.

\section{RESUMEN}

Hoy en día el conejo europeo (Oryctolagus cuniculus) es considerado invasivo en varios países. Sin embargo, los motivos del inicio de la explotación de este lagomorfo en el Paleolítico Superior en la península ibérica son objeto de un intenso debate sin que todavía exista un consenso general sobre las causas que explican su introducción en la dieta de los cazadores-recolectores ibéricos. En este artículo, primero presentamos cómo su caza comenzó antes del Último Máximo Glacial (UMG), aumentando durante este perio-

\footnotetext{
* Cette recherche a été financée par le Conseil de recherches en sciences humaines du Canada, sous la subvention \#435-2016-1158, et le Fonds de Recherche du Québec-Société et Culture sous la subvention \#SE-179537 (à Burke).

a Université de Montréal. Faculté des arts et des sciences - Département d'anthropologie. Pavillon Lionel-Groulx local C3055. CP 6138 Centre-Ville. Montréal. QC H3C 3J8. Canada. Emails: SS samuel.fabien.seuru@umontreal.ca (Auteur correspondant) https://orcid.org/0000-0003-2713-4044 ; AB a.burke@umontreal.ca https://orcid.org/0000-0002-7033-7798

b Université de Montréal. Faculté des arts et des sciences - Département de géographie. Pavillon des Sciences (MIL), local B-5009. 1375 Ave. Thérèse-Lavoie-Roux. Montréal. QC H2V 0B3. Canada. Email: 1.perez@umontreal.ca https://orcid.org/0000-0002-6599-9893

Reçu 1-II-2021; accepté 16-IV-2021.
} 
do. Luego evaluamos las principales hipótesis del Modelo de Amplitud de Dieta (Diet Breadth Model), desarrollado a partir de la Teoría del Forrajeo Óptimo (Optimal Foraging Theory). Este modelo (a veces implícito) es el origen de algunas de estas hipótesis, pero hasta ahora nunca se habia aplicado de forma concreta al UMG en la península ibérica. En consecuencia, nosotros lo hemos hecho y los resultados obtenidos no parecen apoyar las hipótesis que defienden una intensificación de la explotación del medio ambiente. Sugerimos que el aprovechamiento de este taxon en torno al UMG en Iberia podría explicarse más bien por el uso de redes entre varias personas.

Mots clés: Dernier Maximum Glaciaire; Oryctolagus cuniculus; hiatus cynégétique; Modèle Classique du Régime Alimentaire (MCRA); Théorie de l'Approvisionnement Optimal; péninsule ibérique.

Key words: Last Glacial Maximum; Oryctolagus cuniculus; cynegetic hiatus; Diet Breath Model; Optimal Foraging Theory; Iberian peninsula.

Palabras clave: Último Máximo Glacial; Oryctolagus cuniculus; hiato cinegético; Modelo de Amplitud de Dieta; Teoría del Forrajeo Óptimo; península ibérica.

\section{INTRODUCTION}

L'analyse du régime alimentaire des humains dans le passé est un thème majeur des recherches sur les modes de subsistance des chasseurs-cueilleurs, en particulier au Paléolithique supérieur. Au début du Paléolithique supérieur, le spectre faunique consommé par les humains anatomiquement modernes commence à se diversifier, avec l'introduction de petites faunes. En Europe de l'Ouest, cela se traduit par l'introduction de lagomorphes, d'oiseaux, de tortues et de mollusques (e. g., Clark et Straus 1986; Stiner et al. 2000). Particulièrement, dans le SudOuest de l'Europe, le lapin (Oryctolagus) domine le registre archéozoologique de trois régions géographiquement distinctes : le Sud de la France (Cochard et Brugal 2004; Jones 2006), l'Espagne (Villaverde et al. 2010; Cortés-Sánchez et al. 2008; Yravedra et al. 2018, 2019; Rufí et al. 2020) et le Portugal (Hockett et Bicho 2000; Davis 2002; Manne et al. 2012). Cependant, l'exploitation intensive du lapin dans le Sud de la France ne semble généralement pas s'observer avant 13000 AP (LópezMartínez 2008), malgré son exploitation au Paléolithique moyen (Cochard et al. 2012). Au contraire, son exploitation intensive dans la péninsule ibérique débute au Paléolithique supérieur (e.g., Villaverde et al. 1996; Aura et al. 2002; Hockett et Haws 2002; Pérez Ripoll 2004) bien que sa consommation soit localement observée au Paléolithique moyen (Blasco et Peris 2012). Dans ce sens, Morin et collègues (2019) suggèrent que dans le Sud de la France et la péninsule ibérique, l'adoption d'une stratégie de subsistance minimisant le risque, mais aussi l'influence des variations climatiques sur l'abondance des proies de grande taille et des densités locales de lagomorphes, peuvent être de possibles causes de l'introduction des léporidés dans la diète des hominines, dont Néandertal, avant le Paléolithique supérieur. Bien que leur exploitation locale soit moins intense qu'au Paléolithique supérieur, ces auteurs indiquent cependant que la diète des populations de Néandertal pouvait être plus large que ce qui est généralement admis au Paléolithique moyen (Morin et al. 2019).

Afin de justifier l'abondance de Oryctolagus dans les sites archéologiques ibériques autour du Dernier Maximum Glaciaire (DMG), il a été proposé que ce taxon a vraisemblablement joué un rôle clé dans la subsistance des chasseurs-cueilleurs, d'autant plus que la péninsule a probablement servi de refuge glaciaire durant cette période (Taberlet et al. 1998; Sommer et Nadachowski 2006). Diverses hypothèses suggèrent que la pression environnementale (Hesse 1985; Hockett et Haws 2002), la pression cynégétique (Clark et Straus 1986; Straus 1992), la réduction de la mobilité résidentielle (Villaverde et al. 1996; Aura et al. 2002), l'innovation technologique (Hockett et Bicho 2000; Hockett et Haws 2002) et la recherche de la qualité nutritionnelle (Hockett et Haws 2003) sont probablement la cause d'une chasse intensive du lagomorphe en Ibérie. D'autant plus, le registre ethnographique souligne que l'acquisition de petites faunes peut s'inscrire dans des relations socio-culturelles complexes entre individus (Speth 2010; Kelly 2013). Le nombre d'hypothèses démontre l'intensité du débat autour de l'exploitation du lapin dans le passé. C'est une tâche complexe de démêler toutes ces hypothèses et de détailler leurs impacts sur notre compréhension des modes de subsistance des humains préhistoriques. Ceci a eu pour résultat un débat qui stagne depuis quelques années, malgré un rebond récent (Lupo et Schmitt 2020; Morin et al. 2020).

Dans cette étude, nous évaluons les différentes hypothèses avancées pour expliquer le rôle du lapin de garenne (Oryctolagus cuniculus) dans les stratégies de subsistance au cours du DMG dans la péninsule ibérique. Nous nous appuyons sur le Modèle Classique du Régime Alimentaire (MCRA; Diet Breadth Model) issu de la Théorie de l'Approvisionnement Optimal (TAO; Optimal Foraging Theory) afin de les comparer. Ce modèle est en effet à l'origine (parfois implicitement) de plusieurs de ces hypothèses. De plus, cela permet de pouvoir les analyser à partir d'un même cadre théorique, facilitant la comparaison.

\subsection{Cadre géographique et climatique}

Aujourd'hui principalement formée par le Portugal et l'Espagne, la péninsule ibérique abrite une grande

Trab. Prehist., 78, N. ${ }^{\circ} 2$, julio-diciembre 2021, pp. 221-236, ISSN: 0082-5638

https://doi.org/10.3989/tp.2021.12273 
diversité d'environnements. De manière générale, la péninsule est divisée en deux biorégions, soit la biorégion "euro-sibérienne" qui s'étale globalement du Nord-Ouest de la péninsule jusqu'au Nord-Est, et la biorégion "méditerranéenne" occupant le reste du territoire (Jones 2016). Au DMG, cette division s'applique et a conduit à une répartition de la végétation extrêmement variée sur le territoire, allant des forêts de conifères et de feuillus dans la biorégion "méditerranéenne" à des zones steppiques dans la biorégion "euro-sibérienne" (e. g., Villaverde et al. 2019).

\subsection{Cadre chronologique}

Le DMG correspond à une période froide durant laquelle le volume mondial de glace a atteint son maximum (Mix et al. 2001). Il est généralement admis que le DMG se termine à environ 16100 ans AP $(19000$ ans cal AP) (Clark et al. 2009). Dans ce travail, nous utilisons la date de 19500 AP (23000 cal AP) globalement utilisée pour fixer le début du DMG (Mix et al. 2001). Le DMG correspond principalement au Solutréen ( 25500- 21-20000 ans cal AP) (Straus 2018), mais s'étale aussi sur le Gravettien (33000-24000 cal AP) et le Magdalénien inférieur (19000-17000 cal AP). De plus, afin de comparer les comportements cynégétiques des humains avant, pendant et après le DMG, nous avons choisi d'intégrer dans cette étude l'ensemble de la période gravettienne, le Solutréen et le Magdalénien.

\section{SÉLECTION DE SITES}

La sélection des sites s'est faite à partir des bases de données de Jones (2016) et de Fa et collègues (2013). Par la suite, une approche inclusive pour la sélection des sites archéologiques a été menée. Seules les dates C14 (non-calibrées) comprises entre le Gravettien et le Magdalénien ont été considérées. Les sites compris entre 19500-16100 ans AP (23000-19000 ans cal AP) ont été attribués au DMG. Les dates dont la déviation est supérieure à 300 ans ont été écartées. Historiquement, les sites n'ont pas été analysés selon les mêmes techniques de fouille, et les petites faunes préhistoriques n'ont pas souvent été considérées dans les analyses archéozoologiques. Jusque dans les années 1980, les espèces de grande taille étaient prises en compte alors que la petite faune était ignorée pour son supposé trop maigre apport en viande. Il est donc fréquent que le lapin soit absent ou qu'il ait une représentation erronée dans les analyses archéozoologiques de sites fouillés il y a plusieurs dizaines d'années. Dès lors, les assemblages fouillés il y a plus de trente ans présentant une anomalie dans le NISP (Number of Identifed Specimens) du lapin ont été rejetés (e. g., Mallaetes). Nous avons décidé de ne retenir que les assemblages pour lesquels le NISP est disponible pour décrire l'abondance de la totalité des faunes dans les sites archéologiques. Les sites dont seul le MNI (Minimal Numbers of Individuals) était utilisé ont été exclus à cause des problèmes de fragmentation et de conservation différentielles inter-espèce et inter-site qui ne peuvent pas être abordés sans considérer le NISP. Aujourd'hui, de nombreux travaux permettent d'attribuer ou d'écarter l'impact de chaque agent dans l'accumulation de restes de léporidés (e. g., Cochard 2004; Pérez Ripoll 2005; Yravedra 2006; Lloveras et al. 2008a, 2008b, 2009). Dès lors, seuls les sites pour lesquels une analyse taphonomique permettant de conclure une participation anthropique des restes de léporidé est disponible, ont été inclus dans cette étude.

Un total de 24 sites a été retenu (Fig. 1, Annexe). Une fois les sites sélectionnés, nous les avons attribués aux zones proposées par Brugal et Yravedra (2005). Ces derniers ont divisé la péninsule en cinq zones biogéographiques très contrastées selon leur topographie, leurs caractéristiques climatiques et leurs associations fauniques. La zone I correspond au Sud-Ouest de la péninsule, dont l'Estrémadure portugaise et la région de l'Algarve. La zone II concerne la région centrale de l'Ibérie, avec le plateau central (meseta) séparé par le système central. La zone III délimite la bordure méditerranéenne et s'étend de Gibraltar au Sud jusqu'à Valence. Enfin, la zone IV concerne le Nord-Est de la péninsule, incluant la Catalogne et la zone V se rapporte au Nord-Ouest de la péninsule.

Enfin, cette étude s'est focalisée sur l'ensemble des herbivores présents dans les sites archéologiques. Dans un premier temps, le pourcentage relatif de lagomorphe selon les autres herbivores chassés par l'humain, a été comparé par zone. Ensuite, les résultats sont analysés et interprétés à l'aide du MCRA issu de la TAO.

\section{DONNÉES CYNÉGÉTIQUES AUTOUR DU DMG}

Les espèces principales chassées par les humains au cours du DMG (sensu largo) sont les suivantes : Bos/Bison, Cervus elaphus, Capra pyrenaica, Equus caballus, Equus hydruntinus, Equus ferus, Rupicapra ( $R$. rupicapra et $R$. pyreneica), Sus scrofa, Capreolus capreolus et Oryctolagus. Par soucis de comparaison entre les sites, les espèces Equus caballus, Equus hydruntinus et Equus ferus ont été regroupées sous le genre Equus. De même, puisque quelques restes de caprinés ont été seulement identifiés au genre, nous avons réuni le NISP de Capra pyrenaica et de Capra 


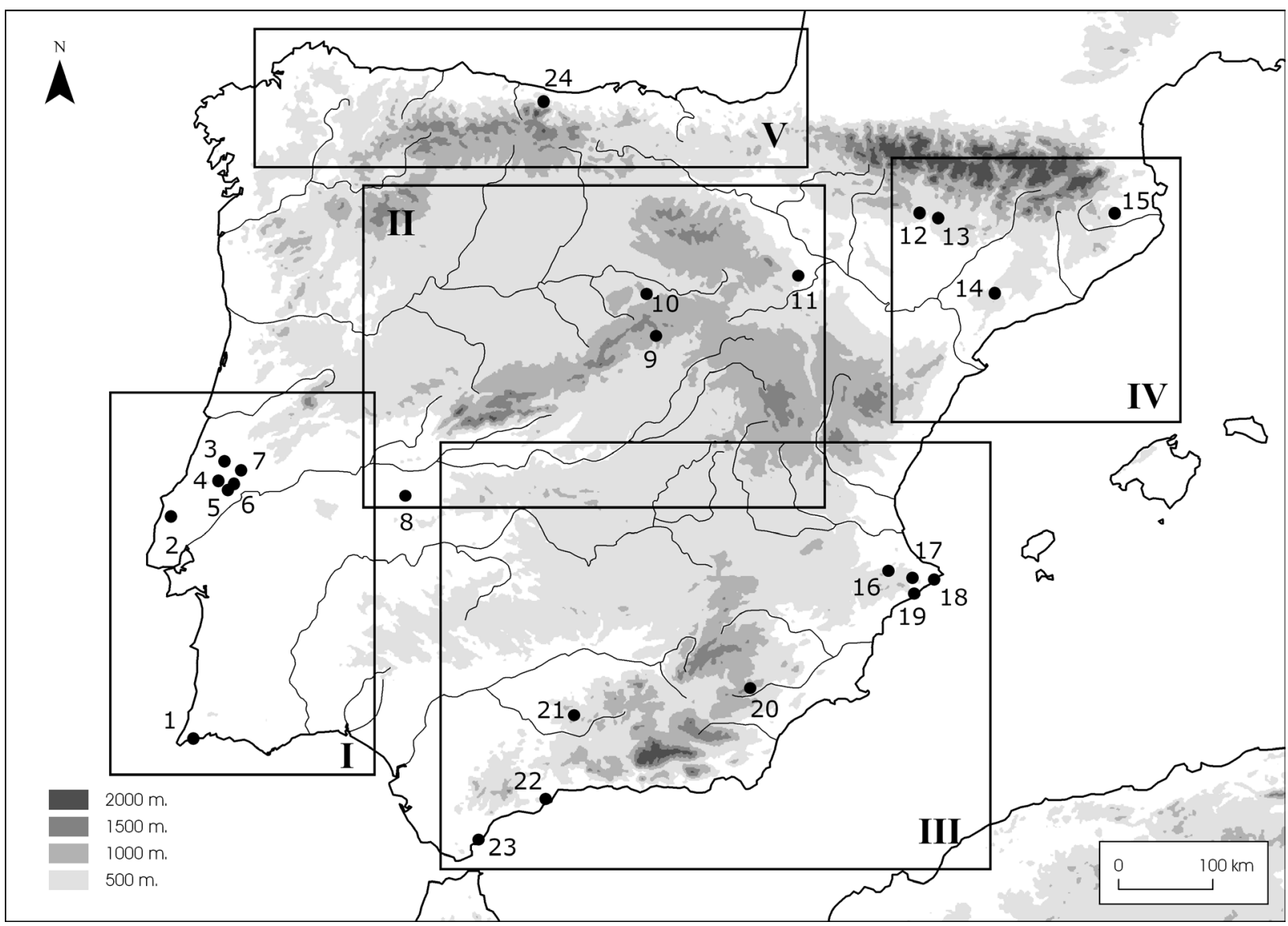

Fig. 1. Répartition des 24 sites retenus dans cette étude (voir Annexe) au sein des cinq zones biogéographiques proposées par Brugal et Yravedra (2005) pour la péninsule ibérique: I. 1. Vale Boi, 2. Suão. 3. Lagar Velho, 4. Lapa do Anecrial, 5 Lapo dos Coelhos, 6. Lapa do Picareiro, 7. Caldeirão. II. 8. Maltravieso, 9. Peña Capón, 10. Peña de Estebanvela, 11. Gato II. IV. 12. Chaves, 13. Peña de las Forcas I, 14. Molí del Salt, 15. Arbreda. III. 16. Beneito, 17. Santa Maira, 18. Les Cendres, 19. la Barriada, 20. Ambrosio, 21. Pirulejo, 22. Nerja, 23. Gorham. V. 24. Coímbre.

$s p$. sous le genre Capra. Ici, la totalité des restes de Oryctolagus se rapporte à l'espèce Oryctolagus cuniculus. Ainsi, pour la suite de l'article, nous nous référons spécifiquement au lapin de garenne (Oryctolagus cuniculus). Finalement, les proportions de chaque espèce dans la figure 2 sont calculées selon le NISP total des principaux mammifères terrestres herbivores exploités mentionnés ci-dessus.

Nos résultats démontrent que la chasse au lapin de garenne a pris de l'ampleur au début du Paléolithique supérieur dans la péninsule ibérique. Une augmentation de l'exploitation du lapin s'observe particulièrement au DMG dans les zones II et III (Fig. 2). Ces résultats sont en accord avec les comportements cynégétiques associés aux humains durant le Paléolithique supérieur ibérique. En effet, une forte exploitation du lapin s'observe à partir du Gravettien et du Solutréen, et elle est maintenue au Magdalénien (Hockett et Bicho 2000; Aura et al. 2002; Davis 2002; Hockett et Haws 2002; Pérez Ripoll 2004;
Riquelme et al. 2005). D'autre part, le lapin est chassé sur l'ensemble de la péninsule, à l'exception de la zone $\mathrm{V}$ où seulement un site a livré des restes du léporidé sur toute la période considérée dans notre étude. La raison de son absence presque totale dans cette zone correspond probablement aux conditions environnementales et climatiques défavorables à son développement, telles que l'humidité de cette région, avec plus de $1000 \mathrm{~mm}$ de précipitations annuelles et l'omniprésence de formations calcaires, défavorables à la création de garennes (Yravedra et al. 2018). Par ailleurs, au DMG, le lapin domine les assemblages fauniques dans les régions favorables aux groupes humains (zones I, III) et aussi dans les environnements plus hostiles (zone II). Nous observons donc une tendance cynégétique généralisée sur l'ensemble du territoire où le lapin domine le spectre de chasse des humains au DMG, à l'exception de la zone V. Autrement dit, pendant le DMG, ce léporidé est exploité en grande quantité dans les régions abritant les 

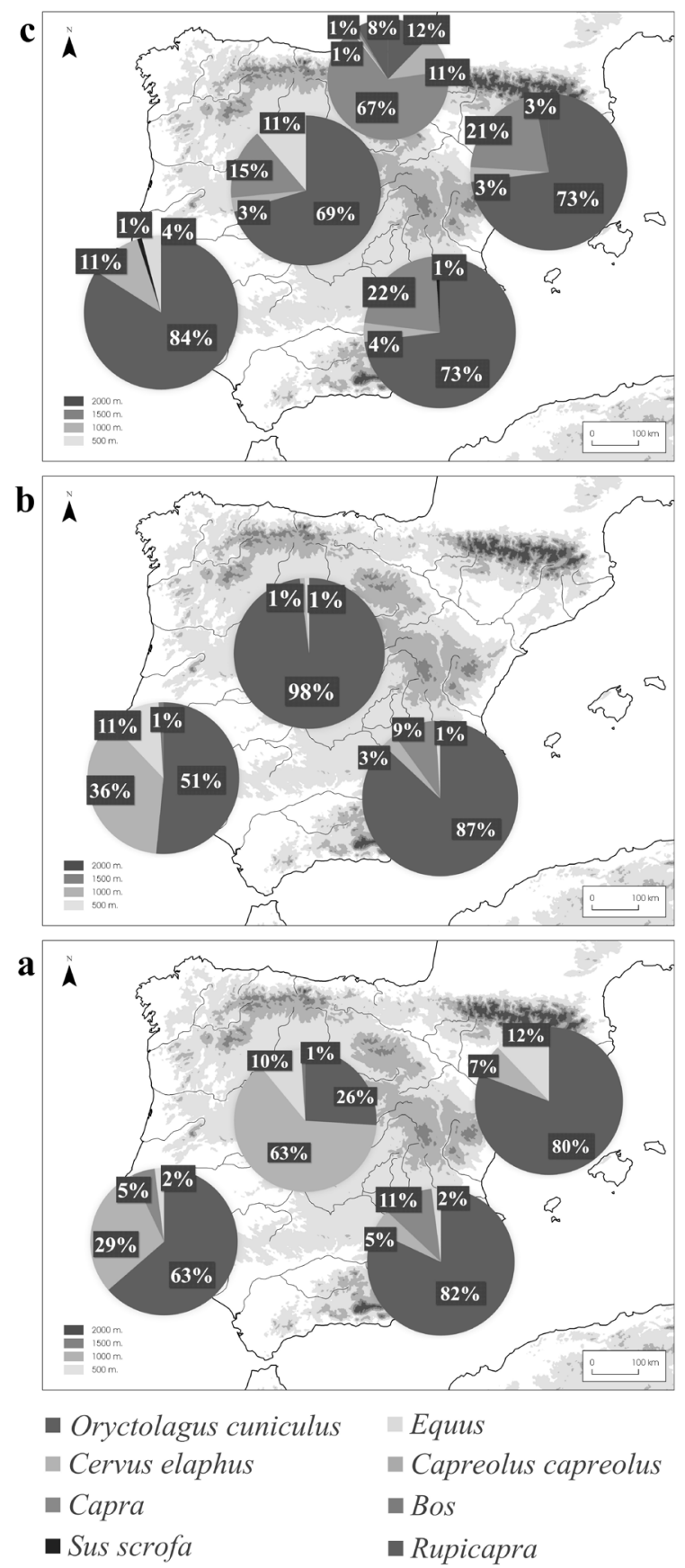

Fig. 2. Proportion (en \%) de Number of Identifed Specimens par zone de chaque espèce au sein du tableau de chasse des humains pour les sites sélectionnés. a: Avant le Dernier Maximum Glaciaire (DMG); b: Au DMG; c: Après le DMG.

plus denses populations humaines mais aussi celles les moins peuplées.

La représentation taxonomique dans chaque site a été sujette à de nombreuses interprétations dont la ma- jorité se base sur la TAO. Notons toutefois que d'autres interprétations sont possibles (e. g., Zeder 2012; Stiner et Kuhn 2016) et ne sont pas forcément exclusives. L'écologie évolutive est un paradigme utile pour interpréter les changements alimentaires dans les séquences archéologiques, notamment en utilisant la TAO. Avant de considérer les différents points de vue sur le statut, le rôle et la signification du lapin dans les sociétés passées à l'aide du cadre théorique de la TAO, il est nécessaire de définir ses principes.

\section{LA THÉORIE DE L'APPROVISIONNEMENT OPTIMAL}

Selon cette théorie appliquée à l'humain, l'exploitation des ressources est faite de manière non aléatoire de la part des chasseurs-cueilleurs afin de répondre à leurs besoins alimentaires, techniques et culturels. Nécessairement, le choix d'une proie répond à des stratégies de subsistance qui témoignent de décisions cohérentes basées sur les diverses ressources potentiellement utilisables. La TAO soutient que les chasseurs-cueilleurs font des choix afin d'optimiser une devise spécifique (currency) (par exemple les calories) en fonction d'un ensemble connu de ressources disponibles, de bénéfices, de coûts et de contraintes de temps. Autrement dit, la TAO affirme que la maximisation du taux net de gain d'énergie va régir les décisions humaines (MacArthur et Pianka 1966). Cette théorie est dérivée de la théorie néo-darwinienne de l'évolution, soit l'application de la sélection naturelle aux problèmes de survie et d'adaptation. La sélection naturelle favorise les stratégies d'acquisition qui augmentent la rentabilité dans l'obtention des ressources. En d'autres termes, la sélection naturelle tend à avantager les individus qui optimisent au mieux les ressources disponibles dans un environnement, selon le rapport énergétique coût/ bénéfice. Dès lors, la TAO est un cadre cohérent constitué d'un ensemble de modèles simples ayant comme objectif de comprendre les raisons pour lesquelles des choix alimentaires sont faits parmi différentes ressources au sein d'un environnement.

\subsection{Le Modèle Classique du Régime Alimentaire (MCRA)}

Parmi les modèles issus de cette théorie, nous utilisons ici le Modèle Classique du Régime Alimentaire (MCRA) (Diet Breadth Model) sur le choix des ressources (MacArthur et Pianka 1966; Bettinger 1991; Cézilly et Benhamou 1996). Ce modèle permet de prédire combien d'espèces doivent être chassées, lesquelles sont délaissées ou la quantité de temps consa- 
crée à l'acquisition de celles-ci (Stephens et Krebs 1986; Bettinger 1991, 2009). Concrètement, le MCRA sert de base (parfois implicitement) à plusieurs des hypothèses citées plus haut pour expliquer l'exploitation intensive du lapin.

Dans le MCRA, dans un premier temps, chaque type de proie est classé selon le retour net d'énergie $\left(e / h_{i}\right)$ calculé à partir du retour énergétique moyen une fois la proie rencontrée $\left(e_{i}\right)$ et de son temps moyen de handling $\left(h_{i}\right)$ (MacArthur et Pianka 1966). Ce temps de handling (ou handling time) correspond au temps d'acquisition qui est la somme du temps consacré à la poursuite, la capture et au traitement de la ressource (Bettinger 1991, 2009). Le temps dédié à la recherche du gibier est donc exclu de l'équation. Dès lors, la ressource possédant le plus grand retour net d'énergie $(e)$ $h_{i}$ ) est celle possédant le plus haut rang dans le classement. A l'inverse, celle possédant le plus petit retour net d'énergie $\left(e / h_{i}\right)$ a le plus bas rang. Pour la suite des calculs, nous avons considéré une exploitation uniquement centrée sur la viande pour le rendement énergétique (excluant la graisse issue des os). Néanmoins, une exploitation des os de l'ensemble de ces herbivores est observée avant, pendant et après le DMG dans la péninsule ibérique (Manne et al. 2012; Yravedra et al. 2018, 2019; Real 2020). Toutefois, nous pensons que l'absence de considération de l'exploitation de la graisse contenue dans les os dans nos calculs n'influence pas la finalité des résultats (les différences de rang en seraient probablement uniquement exagérées). D'autre part, cela permet d'uniformiser nos résultats avec différents travaux ayant appliqué la TAO pour comprendre la diète des humains dans différents contextes du passé (e. g., Hockett et Haws 2003; Henrikson 2004; Byers et Ugan 2005; Jones 2006).

\subsubsection{Calcul du rang des taxons}

A défaut d'informations sur le temps d'acquisition (handling time) dans le passé, la masse de la proie a été conventionnellement utilisée comme proxy pour définir le rang de chaque ressource dans un contexte archéologique. Ceci est connu sous le nom du proxy de masse, ou body-size proxy. Par comparaison, les plus grandes proies procurent une quantité plus importante de nourriture que les petites proies. Les archéologues, par conséquent, ont catégorisé les espèces suivant leur taille : les grandes espèces sont de haut-rang tandis que les petites sont jugées de bas-rang (e.g., Broughton et al. 2011). De même, des espèces de même taille sont de rang égal et sont classées dans le même groupe ( $e$. g., Grayson et Cannon 1999). Néanmoins, l'utilisation du proxy de taille est mise en question par bon nombre d'auteurs et il est sujet à des recalibrations (Stiner et al.
2000; Jones 2006; Lupo et Schmitt 2016, 2020; Morin et al. 2020). Tout d'abord, les coûts liés à la poursuite et au traitement de la proie peuvent être influencés par les caractéristiques de celle-ci. Par exemple, le mécanisme de défense, les caractéristiques physiques ou la vitesse de déplacement de la proie influencent théoriquement la rentabilité de sa chasse (Stiner et al. 2000; Lupo et Schmitt 2020). D'autre part, le rang du lapin est directement influencé par les techniques d'acquisition employées (e. g., chasse collective, utilisation de filets), et donc du temps d'acquisition (Jones 2006; Morin et al. 2020). Conséquemment, il ne faut pas négliger le temps d'acquisition afin d'estimer le rang d'un taxon. Dès lors, nous avons considéré pour l'ensemble des ressources le temps associé à leur poursuite, leur capture et leur traitement puisque le temps d'acquisition peut influencer leur rang. Ainsi, les ressources sont classées selon leur rentabilité, et non pas uniquement selon leur valeur calorique.

Hormis pour le lapin, nous avons utilisé la masse de chaque taxon considéré dans cette étude, obtenue à partir des travaux de Fa et collègues (2013). Nous nous basons sur Morin et collègues (2020) pour la masse moyenne de $1 \mathrm{~kg}$ du lapin de garenne afin de tenir compte des juvéniles. Ensuite, chez les mammifères terrestres, en moyenne, $2,8 \mathrm{~g}$ de graisse sont présents dans $100 \mathrm{~g}$ de viande (basé à partir du cheval, bison, cerf, lapin, sanglier et renne) (Hockett et Haws 2003). De plus, il est généralement accepté que $1 \mathrm{~g}$ de graisse est l'équivalent de $9 \mathrm{Kcal}$ (kilocalories). Le calcul des Kcal à partir de la consommation de la graisse comprise dans la viande d'un individu est donc le suivant : $y=$ masse $* 28 * 9$. Dans le même sens, chez les mammifères terrestres, en moyenne, $22 \mathrm{~g}$ de protéine sont présents dans $100 \mathrm{~g}$ de viande (basé à partir du cheval, bison, cerf, lapin, sanglier et renne) (Hockett et Haws 2003). En outre, il est généralement accepté que $1 \mathrm{~g}$ de protéine est l'équivalent de $4 \mathrm{Kcal}$. Alors, la quantité de Kcal obtenue à partir de la consommation des protéines dans la viande d'un individu est le suivant : $y=$ masse * $220 * 4$. Ensuite, la quantité profitable d'une proie est calculée suivant cette équation (Morin et al. 2021) : y $=-0.0198 x^{0.25}+0.9$ où $x$ est la masse du taxon et $y$ est la quantité profitable. Cela nous permet d'obtenir pour chaque taxon la quantité de Kcal profitables (Tab. 1).

Par la suite, nous utilisons les équations issues des travaux de Miracle ${ }^{1}$ afin d'estimer le temps de traitement et le taux de rencontre. Ainsi, le temps de traitement est calculé suivant l'équation : $y=-0,422( \pm 112)$

${ }^{1}$ P. T. Miracle. Broad-spectrum adaptations re-examined: huntergatherer responses to late glacial environmental changes in the Eastern Adriatic. Dissertation doctorale non-publiée, University of Michigan. 1995.

Trab. Prehist., 78, N. ${ }^{\circ} 2$, julio-diciembre 2021, pp. 221-236, ISSN: 0082-5638

https://doi.org/10.3989/tp.2021.12273 
$+0,444( \pm 0,026) * x$, où $\mathrm{y}$ est le temps de traitement en heures et $x$ la masse du taxon.

Pour les données disponibles concernant le temps de poursuite (incluant la capture), Marín Arroyo (2008) fixe à 30 minutes le temps de poursuite associé à la chasse du cerf et du bouquetin. Nous fixons à 2 heures le temps de poursuite du sanglier, basé sur la comparaison de la chasse du pécari à lèvres blanches par les Ache (Janssen et Hill 2014). Le temps d'au moins 1 heure est fixé pour le cheval. Comparativement, dans un environnement différent, Lupo et Schmitt (2016) ont observé un temps de poursuite du zèbre des plaines (Equus quagga) de 1 heure. Ainsi, le temps d'acquisition est obtenu en additionnant le temps de traitement et de poursuite/capture. Celui du cerf et du bouquetin obtenu est comparable avec les données de Marín Arroyo (2008). A partir des observations de Simms (1987) sur le cerf de Virginie (Odocoileus virginianus) et le mouflon Canadien (Ovis canadensis), Marín Arroyo (2008) fixe le temps d'acquisition à 2 heures pour le cerf et le bouquetin, assimilables à ces deux espèces. Pour les taxons dont le temps de poursuite n'était pas déterminable dans la littérature, nous nous sommes basés sur plusieurs travaux estimant le temps d'acquisition. Pour le chevreuil et le chamois, ce temps est limité à 1,5 heures (Marín Arroyo 2007, cité dans Marín Arroyo 2008). Le total de 9 heures pour les bovinés est issu des observations de Henrikson (2004) pour le bison américain. Un temps de 2 heures (Morin et al. 2020) est attribué au lapin, dont la technique de chasse employée est de creuser la garenne. Dès lors, grâce à l'ensemble de ces résultats, le retour net d'énergie $(e /$ $h_{i}$ ), exprimé en $\mathrm{Kcal} /$ heure $(\mathrm{Kcal} / \mathrm{hr})$, a été calculé pour chaque ressource, ainsi que son rang associé. Ainsi, dans la péninsule et au cours du Paléolithique supérieur, le cheval est la proie de plus haut-rang, suivi du cerf, des bovinés, du bouquetin, du sanglier, du chamois et du chevreuil. Le lapin possède le rang le plus bas (Tab. 1).

Ensuite, la décision d'introduire une proie dans la diète dépend uniquement de sa rentabilité (ou retour net d'énergie $\left.\left(e / h_{i}\right)\right)$ par rapport au Retour Net d'Acquisition $(R N A)$ de l'ensemble des ressources. Pour calculer le $R N A$, le chasseur a besoin de connaitre pour

\begin{tabular}{|c|c|c|c|c|c|c|c|c|}
\hline Taxon & Bos & Cheval & Cerf & Bouquetin & Chevreuil & Chamois & Sanglier & Lapin \\
\hline Masse (kg) & 643 & 400 & 217.75 & 60.2 & 22.5 & 32.8 & 84.5 & 1 \\
\hline Graisse (Kcal) & 162036 & 100800 & 54873 & 15170.4 & 5670 & 8265.6 & 21294 & 244.8 \\
\hline Protéine (Kcal) & 565840 & 352000 & 191620 & 52976 & 19800 & 28864 & 74360 & 846.8 \\
\hline $\begin{array}{c}\text { Total Kcal } \\
\text { (graisse+protéine) }\end{array}$ & 727876 & 452800 & 246493 & 68146.4 & 25470 & 37129.6 & 95654 & 1091.8 \\
\hline Quantité profitable (\%) & 80 & 81 & 82 & 84 & 86 & 85 & 84 & 88 \\
\hline Kcal profitables & 582510 & 367427 & 203097 & 57574 & 21825 & 31657 & 80346 & 961 \\
\hline $\begin{array}{c}\text { Temps de traitement } \\
\text { (processing time) }\end{array}$ & 3.35 & 2.85 & 2.25 & 1.32 & 0.83 & 1 & 1.53 & 0.02 \\
\hline $\begin{array}{c}\text { Temps de poursuite } \\
\text { (pursuit time) }\end{array}$ & $\mathrm{s} / \mathrm{n}$ & 1 & 0.5 & 0.5 & $5 / \mathrm{n}$ & $\mathrm{s} / \mathrm{n}$ & 2 & $\mathrm{~s} / \mathrm{n}$ \\
\hline $\begin{array}{c}\text { Temps total } \\
\mathbf{d} \text { 'acquisition } \\
\text { (handling time) }\end{array}$ & 9 & 3.85 & 2.75 & 1.82 & 1.5 & 1.5 & 3.53 & 2 \\
\hline $\boldsymbol{e}_{\boldsymbol{i}} \boldsymbol{i}$ & 64723 & 95435 & 73854 & 31634 & 14550 & 21122 & 22779 & $1269^{*}$ \\
\hline Rang & 3 & 1 & 2 & 4 & 7 & 6 & 5 & 8 \\
\hline Densité & 0.34 & 4.05 & 2.9 & 2 & 7.61 & 2 & 3.57 & 357.9 \\
\hline $\begin{array}{c}\text { Taux de rencontre } \\
\text { (Individu/heure) }\end{array}$ & 0,000382 & 0,004664 & 0,003314 & 0,002271 & 0,008975 & 0,002271 & 0,004098 & 0,833907 \\
\hline
\end{tabular}

Tab. 1. Estimations du rang des principales espèces exploitées par les chasseurs-cueilleurs dans la péninsule ibérique au Paléolithique supérieur. Temps en heure. $e / h_{i}$ : retour net d'énergie. Les données concernant les Kcal profitables du lapin de garenne sont dérivées de Morin et collègues (2020). * Le retour net énergétique de $1269 \mathrm{Kcal} / \mathrm{hr}$ correspond à la chasse de trois lapins par deux personnes en 1 heure en creusant la garenne avec des pelles. 
chaque ressource son taux de rencontre $\left(r_{i}\right)$, en plus de $e_{i}$ et $h_{i}$. L'utilisation de l'équation de Holling par Stephens et Krebs (1986: 22) met en relation ces variables comme suit :

$$
R N A=\frac{\sum_{i=1}^{n} r_{i} e_{i}}{1+\sum_{i=1}^{n} r_{i} h_{i}} \quad(\text { Équation } 1)
$$

Cette équation calcule donc le $R N A$ cumulatif suivant l'ajout de plus en plus de ressources dans le régime alimentaire des humains. L'introduction des proies commence avec la proie de plus haut-rang, c'est-à-dire avec celle dont le retour net d'énergie $e_{i} / h_{i}$ est le plus grand. Afin de dériver le taux de rencontre, nous avons tout d'abord utilisé les valeurs de densité pour chaque taxon dans la péninsule au Paléolithique supérieur issues des travaux de Fa et collègues (2013). A partir de ces données, le taux de rencontre est calculé selon l'équation suivante : $y=1,12 E-3( \pm 2 E-4) * x+$ $7,81 E-6( \pm 2,2 E-6) * x^{2}$, où $y$ est le nombre d'individu par heure (taux de rencontre) et $x$ et la densité du taxon. L'équation $y=0,00233( \pm 9.6 E-5) * x$, adaptée pour les proies de petite taille, est utilisée pour le taux de rencontre du lapin. Ainsi, le $R N A$ de chaque ressource, exprimé en $\mathrm{Kcal} /$ heure $(\mathrm{Kcal} / \mathrm{hr})$, a été calculé dans le tableau 2.

Lorsqu'une ressource est plus rentable que le $R N A$ calculé en fonction de son ajout dans la diète, alors cette ressource doit être poursuivie. Au contraire, si une ressource rencontrée est moins rentable que cet $R N A$ calculé, elle devrait être ignorée. Autrement dit, si $e$ / $h_{i}>R N A$, alors la ressource devrait être incluse dans le régime optimal; si $e_{i} / h_{i}<R N A$, alors elle devrait être exclue. Le régime alimentaire optimal est ainsi déterminé en introduisant dans l'ordre des ressources de plus basrang jusqu'à ce que $e / h_{i}$ de la prochaine ressource soit inférieur au $R N A$ calculé. La ressource "seuil" à partir de laquelle le régime ne devient plus optimal est très bien visible grâce à une représentation graphique (Fig. 3). Le croisement entre les courbes $R N A$ et $e / h$ indique lorsque le régime n'est plus optimal. La diète optimale des humains en Ibérie durant le Paléolithique supérieur

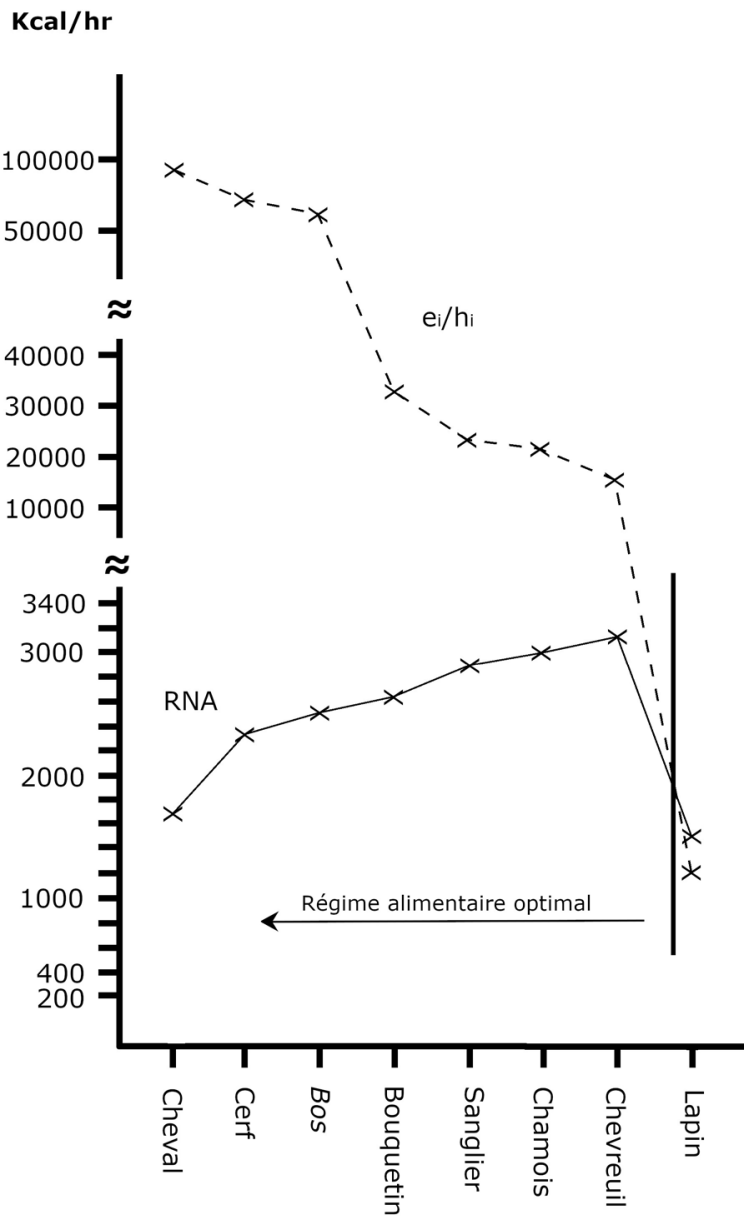

Éléments introduits suivant leur rang

Fig. 3. Courbe du régime alimentaire optimal des populations humaines durant le Paléolithique supérieur en Ibérie. La ligne verticale indique le seuil à partir duquel le régime alimentaire n'est plus optimal.

prédit d'inclure uniquement les proies de plus haut-rang que le lapin. Le lagomorphe est donc exclu de ce régime alimentaire optimal puisque son retour net énergétique

\begin{tabular}{|c|c|c|c|c|c|c|c|c|}
\hline Taxon & Cheval & Cerf & Bos & Bouquetin & Sanglier & Chamois & Chevreuil & Lapin \\
\hline Rang & 1 & 2 & 3 & 4 & 5 & 6 & 7 & 8 \\
\hline RNA * & 1683 & 2324 & 2532 & 2648 & 2925 & 2984 & 3130 & 1514 \\
\hline $\boldsymbol{e}_{\boldsymbol{i}} \boldsymbol{h}_{\boldsymbol{i}}$ & 95435 & 73854 & 64723 & 31634 & 22779 & 21122 & 14550 & 1269 \\
\hline
\end{tabular}

Tab. 2. Estimations du Retour Net d'Acquisition (RNA). Les taxons sont ordonnés en fonction de leur rang dans le Modèle Classique du Régime Alimentaire. * Le RNA est calculé suivant l'équation 1. ei/hi : retour net d'énergie. 
(1269 Kcal/hr) est inférieur au RNA calculé pour les huit taxons considérés ici $(1514 \mathrm{Kcal} / \mathrm{hr})$.

Le retour net énergétique de $1269 \mathrm{Kcal} / \mathrm{hr}$ est associé à un épisode de chasse ayant abouti à l'obtention de trois lapins par deux personnes en 1 heure (Morin et al. 2020). Notons que cette chasse employée pour acquérir trois lagomorphes est la plus rudimentaire de celles exposées dans Morin et collègues (2020), où le principe est de creuser la garenne avec des pelles. Par conséquent, dans le cas où ce type de chasse est employé, le lagomorphe, exclu du régime optimal, est donc une proie de bas-rang. Également, si le cheval est en effet la proie de plus haut-rang, son taux de rencontre par les humains, a une très grande importance sur l'amplitude du régime (et donc l'introduction du lapin dans la diète).

Par la suite, nous avons voulu déterminer l'influence que différentes techniques de chasse du lapin peuvent avoir quant à l'introduction de cette proie dans le régime optimal des humains.

\subsubsection{Calcul du rang associé au lapin selon différentes techniques de chasse}

La dépendance de la masse pour déterminer le rang des espèces ne tient pas compte des techniques de chasse qui peuvent influencer directement sur le temps d'acquisition de la ressource, et donc son rang (Bettinger 1991; Lupo et Schmitt 2016). Autrement dit, ce temps est une variable dépendante du type de chasse associé à l'acquisition de la proie. Le lapin de garenne se regroupe dans les garennes (Villafuerte et Moreno 1997) et son comportement anti-prédation ne représente aucun danger pour l'humain. Dès lors, ces connaissances de l'écologie et de l'éthologie de l'espèce pourraient diminuer la durée de la recherche de la proie, et ainsi influencer son rang. Dans ce sens, Morin et collègues (2020) suggèrent que l'exploitation d'une garenne peut contribuer à des retours énergétiques très grands (se rapprochant à ceux des grandes proies comme le caribou ou l'élan), surtout lorsque celle-ci est densément peuplée. Ces auteurs prennent en compte la pluralité des types de proie au sein d'une même espèce et considèrent qu'une garenne densément peuplée remet en cause le classement par rang des ressources (Morin et al. 2020). Nous avons vérifié ce raisonnement dans notre étude de cas. Ainsi, dans le MCRA, les chasseurs-cueilleurs ne se concentrent plus sur la capture d'un lapin mais plutôt sur la capture d'une garenne. Une garenne abrite en moyenne entre 3 et 20 individus (Villafuerte et Moreno 1997). Puisque la taille d'une garenne est étroitement liée à l'abondance du lapin (Parer et Wood 1986), nous avons divisé le nombre de 357,9 individus par $\mathrm{km}^{2}$ obtenu dans les travaux de
Fa et collègues (2013) et utilisé dans nos précédents calculs de rang, par un nombre d'individus compris entre 3 et 20. Par simplicité dans les calculs, nous supposons ici que les garennes ont toutes un nombre identique d'individus (n'étant pas souvent le cas comme nous en discutons plus bas). Le résultat est une densité comprise entre 119,3 (pour 3 individus par garenne) et 17,9 (pour 20 individus par garenne) garennes par $\mathrm{km}^{2}$ au Paléolithique supérieur ibérique. Nous avons comparé ensuite les rendements énergétiques obtenus dans les récents travaux de Morin et collègues (2020), en fonction de la méthode de chasse associée (Tab. 3). Nous considérons ici que chaque méthode d'exploitation de garenne aboutit toujours au même nombre d'individus capturés. Tout d'abord, nous observons à travers nos résultats que la garenne n'est pas rentable pour les humains lorsqu'elle est exploitée uniquement par creusage (aboutissant à l'acquisition constante de 3 lapins). En effet, l'exploitation d'une garenne selon cette technique n'est pas optimale car son rendement énergétique $e / h_{i}(1269 \mathrm{Kcal} / \mathrm{hr})$ est toujours inférieur au $R N A$ cumulé (Tab. 3 ). Si dans l'environnement, toutes les garennes possèdent un nombre constant d'individus compris entre 3 et 20, l'utilisation du creusage comme technique pour acquérir à chaque fois 3 individus n'est pas rentable. Au contraire, lorsque les humains recherchent une garenne dans le but de l'exploiter à travers l'utilisation de filets, la garenne n'est plus de bas-rang. L'usage de filets se réalise selon le procédé de chasse net driving qui consiste à conduire les lapins dans des filets encerclant la garenne (voir Morin et al. 2020 pour plus de détails). Ainsi, avec une moyenne de 7 lapins capturés en exploitant une garenne selon cette méthode, la garenne entre dans le régime optimal car son rendement énergétique $e / h_{i}(3947 \mathrm{Kcal} /$ hr) est supérieur au $R N A$ cumulé (Tab. 3). Par ailleurs, la densité de garenne observée dans des habitats optimaux peut atteindre entre 10 et 14 garennes par hectare (Gea-Izquierdo et al. 2005; Gálvez-Bravo 2008). Dans certains cas, jusqu'à 60 individus sont présents dans une seule garenne pendant la période de reproduction (Jones 2006). Lors de la rencontre entre des chasseurs et une garenne possédant une telle densité de lapins, le rendement énergétique peut s'élever lors d'une acquisition en masse (Jones 2006). Par exemple, cette méthode de chasse peut procurer un nombre élevé de lapins (35 individus) depuis une seule garenne (Morin et al. 2020). Nous avons testé ici cette hypothèse car un des principes du MCRA est que le choix du chasseur dépend uniquement de l'abondance des ressources de haut-rang. Dès lors, la garenne étant de haut-rang lors de l'utilisation de filets, son abondance influe théoriquement sur le choix du chasseur. Ainsi, une chasse de 35 lapins depuis une seule garenne par 3 hommes pendant 90 minutes et avec l'utilisation de filets, procure 
a.

\begin{tabular}{|c|c|c|c|c|c|c|c|}
\hline Taxon & Cheval & Cerf & Bos & Bouquetin & Sanglier & Chamois & Chevreuil \\
\hline Rang & 1 & 2 & 3 & 4 & 5 & 6 & 7 \\
\hline RNA & 1683 & 2324 & 2532 & 2648 & 2825 & 2984 & 3130 \\
\hline $\boldsymbol{e i} / \boldsymbol{h i}$ & 95435 & 73854 & 64723 & 31634 & 22779 & 21122 & 14550 \\
\hline b.
\end{tabular}

\begin{tabular}{|c|c|c|}
\hline $\begin{array}{c}\text { Garen- } \\
\text { ne de 3 } \\
\text { lapins }\end{array}$ & $\begin{array}{c}\text { Garenne } \\
\text { de 20 } \\
\text { lapins }\end{array}$ & $\begin{array}{c}\text { Garenne } \\
\text { de 60 } \\
\text { lapins }\end{array}$ \\
\hline 8 & 8 & 8 \\
\hline 2222 & 2938 & 3063 \\
\hline 1269 & 1269 & 1269 \\
\hline
\end{tabular}

\begin{tabular}{|c|c|c|c|c|c|c|c|}
\hline Taxon & Cheval & Cerf & Bos & Bouquetin & Sanglier & Chamois & Chevreuil \\
\hline Rang & 1 & 2 & 3 & 4 & 5 & 6 & 7 \\
\hline RNA & 1683 & 2324 & 2532 & 2648 & 2825 & 2984 & 3130 \\
\hline ei/hi & 95435 & 73854 & 64723 & 31634 & 22779 & 21122 & 14550 \\
\hline
\end{tabular}

\begin{tabular}{|c|c|c|}
\hline $\begin{array}{c}\text { Garen- } \\
\text { ne de 7 } \\
\text { lapins* }\end{array}$ & $\begin{array}{c}\text { Garenne } \\
\text { de 20 } \\
\text { lapins }\end{array}$ & $\begin{array}{c}\text { Garenne } \\
\text { de 60 } \\
\text { lapins }\end{array}$ \\
\hline 8 & 8 & 8 \\
\hline 2773 & 2992 & 3083 \\
\hline 3947 & 3947 & 3947 \\
\hline
\end{tabular}

c.

\begin{tabular}{|c|c|c|c|c|c|c|c|c|}
\hline Taxon & Cheval & Cerf & Bos & Bouquetin & Sanglier & Chamois & $\begin{array}{c}\text { Garenne de 35 } \\
\text { lapins }\end{array}$ & Chevreuil \\
\hline Rang & 1 & 2 & 3 & 4 & 5 & 6 & $\mathbf{7}$ & 8 \\
\hline RNA & 1683 & 2324 & 2532 & 2648 & 2825 & 2984 & $\mathbf{2 9 0 7}$ & 3050 \\
\hline $\boldsymbol{e i} / \boldsymbol{h i}$ & 95435 & 73854 & 64723 & 31634 & 22779 & 21122 & $\mathbf{1 9 7 3 7}$ & 14550 \\
\hline
\end{tabular}

d.

\begin{tabular}{|c|c|c|c|c|c|c|c|c|}
\hline Taxon & Cheval & Cerf & Bos & Bouquetin & Sanglier & Chamois & $\begin{array}{c}\text { Garenne de 60 } \\
\text { lapins }\end{array}$ & Chevreuil \\
\hline Rang & 1 & 2 & 3 & 4 & 5 & 6 & 7 & 8 \\
\hline RNA & 1683 & 2324 & 2532 & 2648 & 2825 & 2984 & $\mathbf{2 9 3 9}$ & 3083 \\
\hline $\boldsymbol{e i} / \boldsymbol{h i}$ & 95435 & 73854 & 64723 & 31634 & 22779 & 21122 & $\mathbf{1 9 7 3 7}$ & 14550 \\
\hline
\end{tabular}

Tab. 3. Comparaison du rang de la garenne en fonction de la méthode de chasse choisie. a. Creusage: 3 lapins acquis; b. Conduite par filet (moyenne) : 7 lapins acquis; c. Conduite par filet (élevé) : 35 lapins acquis; d. Conduite par filet (élevé) : 35 lapins acquis. RNA : Retour Net d'Acquisition. ei/hi : retour net d'énergie. * Cette chasse est associée à l'acquisition de 7 lapins issus de la même garenne grâce à la participation de trois hommes pendant 90 minutes (Morin et al. 2020). Puisque cette chasse est associée à l'acquisition de 7 lapins, nous avons effectué nos calculs avec un minimum de 7 individus par garenne au lieu de 3 .

un rendement énergétique $e_{i} / h_{i}$ s'élevant à $19737 \mathrm{Kcal} /$ hr (graisse extramusculaire exclue) (Morin et al. 2020). Par conséquent, dans le cas où toutes les garennes sont composées par au minimum 35 et 60 individus et que 35 individus sont toujours obtenus, alors le classement de la garenne en serait modifié, arrivant à la $7^{\text {ème }}$ place, devant le chevreuil. Néanmoins, une telle densité constante de lapin au sein de chaque garenne n'est pas réaliste. En effet, les garennes densément peuplées avec plus de 35 lagomorphes par vivier n'existent que localement, dans les habitats optimaux. Dans ce sens, une connaissance étroite de la localisation de ce type de garenne densément peuplée dans le passé ainsi que de l'éthologie de ce taxon, couplée à l'utilisation de filets par plusieurs personnes, a probablement permis l'exploitation du grand nombre de lapin observée au Paléolithique supérieur ibérique.

\section{DISCUSSION}

La figure 4 compare le registre archéozoologique du Paléolithique supérieur en Ibérie (colonne de droite) avec le classement par rang des espèces suivant leur rentabilité déterminée traditionnellement selon la masse (Fig. 4a), et selon la masse et le temps d'acquisition (Fig. 4b). Très clairement, le classement par rang des espèces diffère lorsque le temps d'acquisition est pris en compte. D'autre part, dans les deux cas, lorsque ce classement est comparé avec le registre archéozoologique, on observe que certaines espèces censées être exploitées ne le sont pas. Plus important encore, le lapin est chassé alors qu'il n'est pas supposé l'être selon l'application du MCRA (sans utilisation de filets par plusieurs personnes). Nous définissons ici le terme de "hiatus cynégétique" (Fig. 4) comme l'absence des

Trab. Prehist., 78, N. ${ }^{\circ}$ 2, julio-diciembre 2021, pp. 221-236, ISSN: 0082-5638

https://doi.org/10.3989/tp.2021.12273 

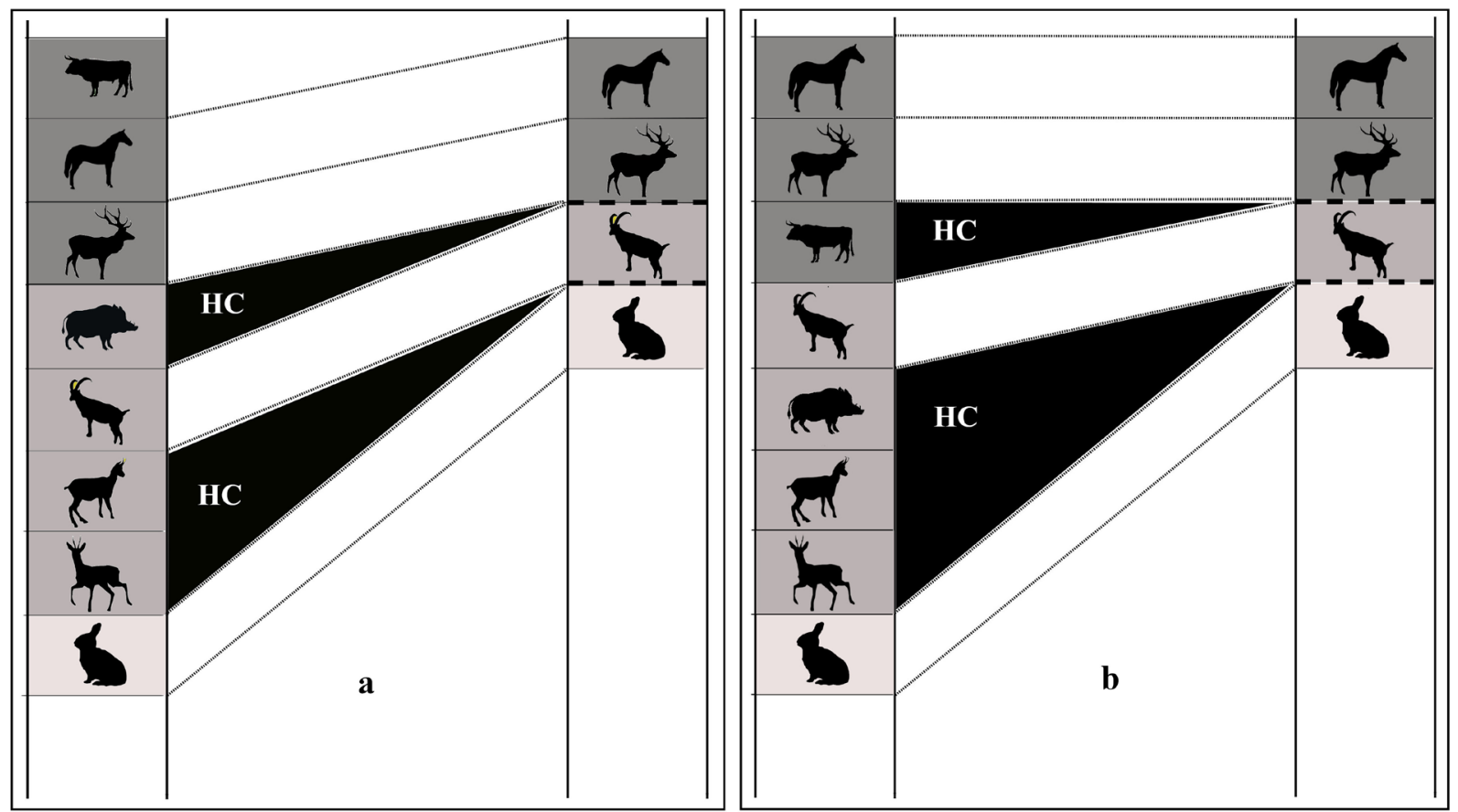

Fig. 4. Comparaison du spectre faunique avec le registre archéozoologique autour du Dernier Maximum Glaciaire (DMG). Colonne de gauche: rang des taxons. Colonne de droite: synthèse des principales proies terrestres chassées par les humains en Ibérie dans les zones biogéographiques de la péninsule ibérique (Brugal et Yravedra 2005) I, II, III et IV. HC: Hiatus Cynégétique. Ligne discontinue: contact anormal dû au HC. a. Utilisation du proxy de taille dans le Modèle Classique du Régime Alimentaire appliqué à la péninsule ibérique autour du DMG. Du haut vers le bas: Proies de grande taille (Bos (auroch), Equus (équidés), Cervus elaphus (cerf)); proies de taille moyenne (Capra (bouquetin), Sus scrofa (sanglier), Rupicapra (chamois ou isard), Capreolus capreolus (chevreuil)); proie de petite taille (Oryctolagus cuniculus (lapin)). b. Utilisation du rang calculé pour chaque proie dans le Modèle Classique du Régime Alimentaire appliqué à la péninsule ibérique autour du DMG.

proies supposées être introduites dans le régime alimentaire lorsqu'il n'est plus rentable de chasser uniquement les proies de plus haut-rang. Concrètement, cela se caractérise notamment par la présence anecdotique générale des espèces de taille moyenne telles que le sanglier, le chamois et le chevreuil dans le tableau de chasse des humains. En effet, ceux-ci ne semblent pas exploités de manière régulière par les humains. Les conséquences du hiatus cynégétique en Ibérie sont l'exploitation du lapin, proie de bas-rang, aux côtés des proies de haut-rang, telles que les équidés, le cerf et le bouquetin.

C'est ce phénomène de hiatus cynégétique qui est à l'origine des diverses hypothèses proposées par la communauté scientifique.

\subsection{Les différentes hypothèses majeures avancées pour expliquer la chasse du lapin de garenne en Ibérie}

Plusieurs hypothèses ont été proposées pour expliquer l'introduction du lapin dans la diète des hu- mains observée avant le DMG dans la péninsule, notamment dans le cadre de l'hypothèse de la Broad Spectrum Revolution (BSR) basée sur les travaux de Flannery (1969). Le raisonnement initial du BSR est que la diversification du régime alimentaire des humains à la fin du Pléistocène dans l'Ouest de l'Asie met en évidence une intensification de l'exploitation de l'environnement. Depuis, le cadre géographique de l'application du $B S R$ a été élargi pour inclure l'Asie et l'Europe (Stiner 2001; Zeder 2012).

Le premier argument majeur du BSR explique qu'une pression cynégétique des humains liée à une augmentation démographique de leur population a entrainé une diversification de la diète (Binford 1968; Flannery 1969; Winterhalder 1981; Stiner 2001; Stiner et Munro 2002). Dans la péninsule ibérique, Straus (2013: 111) se base en partie sur cet argument et définit ce phénomène de "situational specialization and overall diversification", caractérisant une intensification de l'exploitation des ressources au Solutréen. En effet, une spécialisation cynégétique envers le cerf et/ou le bouquetin, devient évidente sur l'ensemble de la péninsule au Solutréen (Straus 1987, 1992, 2018; Aura et al. 
2002; Marín Arroyo 2009). En même temps, une diversification du régime alimentaire des humains se produit avec l'introduction de petites proies dont le lapin dans toute la péninsule, excepté le Nord-Ouest. Néanmoins, plutôt que l'augmentation démographique de la population humaine au DMG, c'est l'augmentation de la densité humaine en Ibérie durant cette période qui serait la cause de cette pression cynégétique envers les proies de grande taille (Clark et Straus 1986). Durant le DMG, les conditions climatiques extrêmes dans le Nord de l'Europe ont probablement entraîné un déplacement des populations humaines et animales au Sud $\mathrm{du} 49^{\circ}$ parallèle Nord (Verpoorte 2009), vers la région méditerranéenne (Jochim 1987). L'intensification de l'exploitation des ressources dans la péninsule est probablement la conséquence d'une pression cynégétique envers les communautés d'herbivores causée par un déséquilibre entre l'arrivée des humains et les grands ongulés sur un territoire limité (Clark et Straus 1986; Straus 1992; Davis 2002).

La seconde hypothèse majeure contraignant les chasseurs-cueilleurs d'élargir leur spectre alimentaire est la pression environnementale (Hesse 1985; Hockett et Haws 2002; Manne et al. 2012). En Ibérie, l'intensification de l'exploitation des ressources avant le DMG à partir du Gravettien peut être liée aux conditions environnementales et climatiques de chaque biorégion. Celles-ci conditionnent la disponibilité des ressources et ont pu modifier les communautés de mammifères et leur taux de rencontre avec les humains.

Par ailleurs, une troisième hypothèse est avancée dans le versant méditerranéen, où plusieurs auteurs ont proposé que la spécialisation cynégétique envers le cerf et le bouquetin est accompagnée d'une réduction de la mobilité résidentielle documentée à la fin du Paléolithique supérieur (Villaverde et al. 1996; Aura et al. 2002; Pérez Ripoll et Martínez Valle 2001). Cette stratégie s'adapte mais est aussi limitée par les déplacements et les migrations saisonnières du cerf et du bouquetin (Aura et al. 2002). Selon ce schéma, les ressources statiques et abondantes dans l'environnement telles que le lapin sont alors revalorisées (Pérez Ripoll 2004). La réduction de la mobilité résidentielle serait le résultat d'un environnement précis, côtier et terrestre aux ressources diverses et riches (Villaverde et al. 1996; Aura et al. 2002). Elle ne représente donc pas une intensification généralisée de l'exploitation des ressources par les humains à cette période (Aura et al. 2002). "Dépendants" de ce schéma de mobilité, l'abondance durant toute l'année du lagomorphe a pu favoriser son exploitation intensive par les humains.

Ensuite, la complexité de la compréhension du régime alimentaire des humains dans le passé s'accroît avec l'ajout d'un argument : la qualité nutritionnelle. Selon la théorie Nutritional Ecology, une diète diver- sifiée permet d'obtenir un plus large éventail de nutriments essentiels (lipides, glucides, protéines, vitamines, minéraux et eau) (Hockett et Haws 2003, 2005). Grâce à une consommation diversifiée et équilibrée, l'espérance de vie des humains augmente et la mortalité infantile diminue (Hockett et Haws 2003, 2005). Le lapin est une source riche de niacine (vitamine PP), phosphore et potassium qui sont des éléments importants pour la croissance (Hockett et Bicho 2000). Ainsi, son exploitation intensive peut être justifiée par la recherche de ces éléments nutritionnelles.

Par ailleurs, tout comme ce dernier argument optimisant la qualité nutritionnelle, il est important de garder en mémoire que les sociétés du passé ont pu chasser pour répondre à des besoins autres qu'énergétiques. Dans ce sens, l'optimisation des relations sociales et des normes culturelles a pu être un objectif qu'il faut prendre en compte lorsque nous analysons le régime alimentaire des groupes humains. La division sexuelle des taches, la dimension du groupe, son organisation interne, sa hiérarchie, son degré de cohésion dans les stratégies cynégétiques et le gain non-alimentaire $(e$. $g$., la fourrure ou la quête de prestige social de certains membres) sont des facteurs à considérer (e.g., Hawkes et al. 1993; Lupo et Schmitt 2002; Brugal 2006; Kuhn et Stiner 2006; Bliege Bird et Bird 2008; Codding et al. 2010; Speth 2010; Real 2020).

Enfin, un argument supplémentaire avancé pour expliquer l'intensification de l'exploitation du lapin est le développement d'innovations technologiques facilitant son acquisition. Ces innovations, comme le filet ou le collet, ont probablement augmenté les chances de capture du lapin (Hockett et Bicho 2000; Aura et al. 2002; Hockett et Haws 2002; Lupo et Schmitt 2002; Stiner et Munro 2002; Jones 2006). Cependant, l'utilisation de telles technologies est difficile à confirmer à travers le registre fossile ibérique, malgré la possible représentation de filet dans l'art pariétal (Straus 1992). Nous discutons l'apport de nos résultats dans le cadre de cette hypothèse ci-dessous.

\subsection{L'hypothèse de l'innovation technologique}

L'hypothèse appuyant l'innovation technologique comme moteur de l'exploitation intensive du lapin, demande de préciser le calcul du profit énergétique associée à la chasse d'une proie à l'aide de différentes technologies. Des changements dans la durée associée à la recherche d'une proie ou de sa poursuite, capture et traitement, permettent de faire des prédictions sur les changements de la diète (Winterhalder 1981). Le classement des espèces est défini par son rang, défini lui-même par le profit énergétique net lié à sa consommation. Si une technologie diminue les coûts cynégé- 
tiques et permet d'économiser du temps dans la chasse au lapin, alors le profit sera plus important, et par conséquent le rang du lapin augmentera. Ainsi, les circonstances de la capture, dont la technologie utilisée dans la chasse, influencent le profit des ressources et donc leur rang (Bettinger 1991; Lupo et Schmitt 2020). Nous avons vérifié ce raisonnement avec la comparaison de différentes techniques d'exploitation du lapin. Finalement, nous sommes d'accord avec Morin et collègues (2020) qui suggèrent premièrement que la pluralité des types de proie devrait être prise en compte dans le calcul du rang. Dans cette étude, un changement dans la détermination du type de ressource (c'est-à-dire une garenne à la place du lapin lui-même) nous a permis d'évaluer les différentes méthodes de chasse employées pour exploiter une garenne. Ce changement dans la définition du type de proie, a des conséquences sur le rang des ressources disponibles dans l'environnement. En effet, la garenne devient une ressource optimale si elle est exploitée à l'aide de filet par plusieurs personnes, sans qu'une acquisition en masse soit nécessaire (plus de 35 individus capturés).

Ces observations sont pertinentes dans la compréhension des causes expliquant l'exploitation du lapin autour du DMG dans la péninsule ibérique. Le changement du type de proie de "lapin" à "garenne" dans le MCRA a permis de relever plusieurs points importants. Nous observons que l'élévation du rang du type de proie "garenne" dépend de la méthode de chasse associée à son exploitation. Si nous considérons la garenne comme type de proie et comparons son rendement énergétique aux autres gibiers chassés, celle-ci est toujours de bas-rang (et est donc exclu du régime optimal) lorsqu'elle est exploitée selon la méthode de creusage et par plusieurs individus. Ceci démontre que la participation de divers individus dans la chasse au lapin ne peut pas expliquer à elle-seule l'exploitation du lagomorphe à partir du MCRA. En revanche, lorsque le procédé de net driving est utilisé par plusieurs personnes afin de capturer ces proies depuis une seule garenne, celle-ci augmente son rang et fait alors partie de la diète optimale. Dans le cas d'une acquisition en masse de lapins (minimum 35 individus) depuis des garennes densément peuplées, le rang augmente encore d'une place. Par conséquent, nous suggérons ici que l'utilisation de filets par plusieurs individus à cette période est une hypothèse plausible pour expliquer l'exploitation en grande quantité du lapin. La matrice élaborée par Driver (1995) combinant l'organisation sociale du groupe avec le mode d'obtention des proies, appuie cette hypothèse. En effet, dans cette matrice, une acquisition en grande quantité d'une proie peut se réaliser lorsque plusieurs personnes d'un groupe collaborent à travers l'utilisation de système de piégeage (tel que le procédé de driving). Ainsi, ce n'est pas l'acquisition en masse qui détermine l'entrée de la garenne dans le régime optimal des humains comme le suggère Jones (2006), mais plutôt l'usage de filets et la complémentarité de divers individus dans la chasse. Son exploitation ne serait ainsi pas liée à une intensification des ressources puisque la garenne est incluse dans le régime optimal des humains. Toutefois, même s'il est en général admis qu'une grande quantité de lagomorphes dans un assemblage implique l'usage de piège dans des zone privilégiées telles que la garenne (Brugal 2006), il reste difficile d'affirmer l'utilisation de telles techniques de chasse dans le passé. Bien qu'il soit rare de retrouver des traces de l'utilisation de filets dans le passé, la technologie à base de fibres a toutefois été utilisée pendant le Paléolithique supérieur en Europe (Adovasio et al. 1997). Ceci démontre que l'utilisation de telles technologies ne dépasse pas les capacités cognitives des humains anatomiquement modernes au Paléolithique supérieur. Notons par ailleurs que les données issues de Morin et collègues (2020) sur le rendement énergétique de l'exploitation de différents types de garenne suivant différentes techniques, ne prennent pas en compte le coût de la maintenance et de la fabrication des technologies utilisées pour capturer les lapins. Or, Lupo et Schmitt (2002) suggèrent que les coûts énergétiques associés à la fabrication des filets et des collets et de leur entretien ne sont pas rentables car ils sont trop élevés par rapport aux bénéfices liés à l'acquisition de petites proies. Toutefois, l'acquisition en masse du lapin permettrait d'augmenter les bénéfices et ainsi de compenser les coûts associés à la manufacture et le maintien de technologies de chasse tels les filets (Ugan 2005; Jones 2006). Par conséquent, il serait nécessaire dans le futur d'inclure ces coûts afin d'obtenir une vision plus nuancée du rang de la garenne (et indirectement du lapin) car ce type de garenne densément peuplée permettant une chasse en masse ne correspond pas au nombre moyen d'individus par garenne (entre 3 et 20 lapins). De même, nous suggérons notamment d'inclure la distribution discrète du nombre d'individus par garenne afin d'obtenir une vision plus réaliste du rang. Nos calculs considèrent que les garennes sont toutes réparties selon le même nombre d'individus par garenne. Dès lors, nous encourageons d'intégrer dans les calculs d'exploitation des ressources cette répartition discontinue dans les garennes puisque celles-ci peuvent être densément peuplées localement (dans les habitats optimaux).

\section{CONCLUSION}

L'utilisation du MCRA révèle que plusieurs hypothèses émises afin d'expliquer le hiatus cynégétique autour du DMG ne semblent pas cohérentes avec ce 
cadre théorique. Les hypothèses de la pression cynégétique et environnementale ainsi que la réduction de la mobilité résidentielle ont été proposées afin d'expliquer l'exploitation intensive du lapin en Ibérie. Nous suggérons ici que la présence du hiatus cynégétique contredit ces hypothèses, puisque l'ordre "d'introduction" des espèces dans la diète des humains suivant leur rang n'est pas respecté. D'autant plus, il s'agit ici de la chasse d'une proie de bas-rang (le lapin) à la place d'espèces de haut-rang (sanglier, chamois et chevreuil). Plutôt, il semble que l'utilisation de technologies comme les filets par plusieurs individus soit une hypothèse valide expliquant l'exploitation du lapin de garenne autour du DMG dans la péninsule ibérique.

Dès lors, nous relevons trois points. Le premier est que la devise (currency) appliquée dans la TAO ne doit pas nécessairement se limiter à l'apport énergétique. Dans ce sens, l'application d'une autre devise, soit la recherche d'une diversité de nutriments, ne contredit pas nos résultats. Ensuite, le choix du type du ressource au sein du MCRA ne doit pas uniquement se limiter au rang taxonomique (exemple Cervus elaphus). Dans notre cas, l'adaptation du type de ressource de Oryctolagus cuniculus à la garenne nous a permis de nuancer les causes de la chasse du lapin. Ici, c'est parce que la garenne est associée à un type de chasse particulier (chasse collective (réalisée par des hommes) et avec des filets) que le changement du type de ressource de "lapin" à "garenne" a influencé son rang.

Finalement, le dernier point est qu'il ne faut pas ignorer toutes les variables capables d'influencer le rang d'une ressource (e. g., Lupo et Schmitt 2002, 2016, 2020; Stiner et Munro 2002; Jones 2006; Lupo 2007). Pour cela, il faut donc que l'interprétation de l'exploitation du lapin de garenne soit caractérisée selon les circonstances de sa chasse autour du DMG. Dans le but d'identifier ces circonstances, il est primordial de prendre en compte tous les facteurs impactant les coûts et les bénéfices durant cette période, incluant les facteurs socio-culturels. En outre, ceux-ci ont probablement joué un rôle dans la présence élevée du léporidé dans la diète des humains autour du DMG. Pour les futures recherches, la vérification de leurs impacts sur l'exploitation du lapin de garenne autour du DMG dans la péninsule ibérique représente un vrai défi si nous voulons comprendre les retombées que sa chasse a eu sur notre espèce.

\section{REMERCIEMENTS}

Nous souhaitons remercier les membres du Groupe de Recherche sur la Dispersion des Hominines (GRDH) pour leurs recommandations. Nous remer- cions également les deux relecteurs anonymes pour leurs commentaires pertinents.

\section{ANNEXE: FICHIER SUPPLÉMENTAIRE}

L'édition électronique de cet article, disponible en libre accès sur le site web de la revue, inclut le tableau : Liste des niveaux sélectionnés dans cette étude et proportion (\% Number of Identified Specimens) de chaque taxon.

\section{BIBLIOGRAFÍA}

Adovasio, J. M.; Hyland, D. C. et Soffer, O. 1997: “Textiles and cordage: A preliminary assessment". In J. Svoboda (ed.): Pavlov I-Northwest. The Upper Paleolithic burial and its settlement context. Dolni Vestonice Studies 4, Academy of Sciences of the Czech Republic. Brno: 403-424.

Aura, J. E.; Villaverde, V.; Pérez, M.; Martinez, R. et Calatayud, P. 2002: "Big game and small prey: Paleolithic and Epipaleolithic economy from Valencia (Spain)". Journal of Archaeological Method and Theory 9 (3): 215-268.

Bettinger, R. L. 1991: "Hunter-gathers as optimal foragers". In R. L. Bettinger, R. Garvey et Sh. Tushingham (eds.): Hunter-gatherers: archaeological and evolutionary theory. Plenum Press. New York: 83-130.

Bettinger, R. L. 2009: "How to calculate Optimal Diet Breadth". In R. L. Bettinger (ed.): Hunter-gatherer foraging: five simple models. Eliot Werner Publications. New York: 1-47.

Binford, L. R. 1968: "Post-Pleistocene adaptations". In S. R. Binford et L. R. Binford (eds.): New Perspectives in Archaeology. Aldine. Chicago: $313-341$

Blasco, R. et Peris, J. F. 2012: “A uniquely broad spectrum diet during the Middle Pleistocene at Bolomor Cave (Valencia, Spain)". Quaternary International 252: 16-31. https://doi.org/10.1016/j.quaint.2011.03.019

Bliege Bird R. et Bird D. W. 2008: "Why women hunt: Risk and contemporary foraging in a western desert aboriginal community". Current Anthropology, 49: 655-693. https://doi.org/10.1086/587700

Broughton, J. M.; Cannon, M. D.; Bayham, F. E. et Byers, D. A. 2011: "Prey body size and ranking in zooarchaeology: theory, empirical evidence, and applications from the northern Great Basin". American Antiquity 76 (3) : 403-428. https://doi.org/10.7183/0002-7316.76.3.403

Brugal, J.-P. 2006: "Petit gibier et fonction de sites au Paléolithique supérieur. Les ensembles fauniques de la grotte d'Anecrial (Porto de Mos, Estremadure, Portugal)". PALEO. Revue d'archéologie préhistorique (18): 45-68. https://doi.org/10.4000/paleo.140

Brugal, J.-P. et Yravedra, J. 2005: "Essai sur la biodiversité des associations de grands mammifères à la fin du Pléistocène dans le Sud-Ouest de l'Europe". Munibe Antropologia-Arkeologia 57: 139-162.

Byers, D. A. et Ugan, A. 2005: "Should we expect large game specialization in the late Pleistocene? An optimal foraging perspective on early Paleoindian prey choice". Journal of Archaeological Science 32 (11): 1624-1640. https://doi.org/10.1016/j.jas.2005.05.003

Cézilly, F. et Benhamou, S. 1996: "Les stratégies optimales d'approvisionnement". Revue d'Ecologie la Terre et la Vie 51: 43-86.

Clark, G. A. et Straus, L. G. 1986: "Synthesis and conclusions. Part I: Upper Paleolithic and Mesolithic hunter-gatherer subsistence in northern Spain”. In La Riera Cave: Stone Age hunter-gatherer adaptations in northern Spain. Anthropological Research Papers 36. Arizona State University. Tempe: 351-366.

Clark, P. U.; Dyke, A. S.; Shakun, J. D.; Carlson, A. E.; Clark, J.; Wohlfarth, B.; ... et McCabe, A. M. 2009: "The last glacial maximum". Science 325 (5941): 710-714. https://doi.org/10.1126/science. 1172873

Cochard, D. 2004: "Influence de l'âge des proies sur les caractéristiques des accumulations de léporidés produites par le hibou grand-duc". In

Trab. Prehist., 78, N. ${ }^{\circ}$ 2, julio-diciembre 2021, pp. 221-236, ISSN: 0082-5638

https://doi.org/10.3989/tp.2021.12273 
J.-P. Brugal et J. Desse (eds.): Petits animaux et sociétés humaines. Du complément alimentaire aux ressources utilitaire. Actes XXIV Rencontres Internationales d'Archéologie et d'Histoire d'Antibes 2004. Éd. APDCA. Antibes: 313-316.

Cochard, D. et Brugal, J.-P. 2004: "Importance des fonctions de sites dans les accumulations paléolithiques de léporidés". In J.-P. Brugal et J. Desse (eds.): Petits animaux et sociétés humaines. Du complément alimentaire aux ressources utilitaire. Actes XXIV Rencontres Internationales d'Archéologie et d'Histoire d'Antibes 2004. Ed. APDCA. Antibes: 283-296.

Cochard, D.; Brugal, J.-P.; Morin, E. et Meignen, L. 2012: "Evidence of small fast game exploitation in the Middle Paleolithic of Les Canalettes Aveyron, France". Quaternary International, 264: 32-51. https://doi.org/10.1016/j.quaint.2012.02.014

Codding, B. F; Bird, D. W. Bird, R. B. 2010: "Interpreting abundance indices: some zooarchaeological implications of Martu foraging". Journal of Archaeological Science 37 (12): 3200-3210. https://doi.org/10.1016/j.jas.2010.07.020

Cortés-Sánchez, M.; Morales-Muñiz, A.; Simón-Vallejo, M. D.; BergadáZapata, M.; Delgado-Huertas, A.; López-García, P.; ...et Vera-Peláez, J. L. 2008. "Palaeoenvironmental and cultural dynamics of the coast of Málaga (Andalusia, Spain) during the Upper Pleistocene and Early Holocene". Quaternary Science Review 27: 2176-2193.

https://doi.org/10.1016/j.quascirev.2008.03.010

Davis, S. J. 2002: "The mammals and birds from the Gruta do Caldeirão, Portugal". Revista Portuguesa de Arqueologia, 5 (2): 29-98.

Driver, J. C. 1995: "Social hunting and multiple predation". In D. V. Campana (ed.): Before farming: hunter-gatherer society and subsistence. MASCA Research Papers in Science and Archaeology 12 (Suppl.). The University Museum, University of Pennsylvania. Philadelphia: 23-38.

Fa, J. E.; Stewart, J. R.; Lloveras, L. et Vargas, J. M. 2013: "Rabbits and hominin survival in Iberia". Journal of human evolution 64 (4): 233241. https://doi.org/10.1016/j.jhevol.2013.01.002

Flannery, K. 1969: "Origins and ecological effects of early domestication in Iran and the Near East”. In P. J. Ucko et G. W. Dimbleby (eds.): The domestication and exploitation of plants and animals: proceedings of a meeting of the Research Seminar in Archaeology and Related Subjects held at the Institute of Archaeology. London University. Aldine Publishing. Chicago: 73-100.

Gálvez-Bravo, L. 2008: El conejo europeo (Oryctolagus cuniculus) como especie ingeniera de ecosistemas. Tesis doctoral. Universidad de Alcalá. http://hdl.handle.net/10017/2182

Gea-Izquierdo, G.; Muñoz-Igualada, J. et San Miguel-Ayanz, A. 2005: "Rabbit warren distribution in relation to pasture communities in $\mathrm{Me}$ diterranean habitats: consequences for management of rabbit populations". Wildlife Research 32 (8): 723-731. https://doi.org/10.1071/WR04129

Grayson, D. K. et Cannon, M. D. 1999: "Human paleoecology and foraging theory in the Great Basin". In Ch. Beck (ed.): Models for the millennium: Great Basin anthropology today. University of Utah Press. Salt Lake City: 141-151.

Hawkes, K.; Altman, J.; Beckerman, S.; Grinker, R. R.; Harpending, H.; Jeske, R. J.; ... et Yellen, J. E. 1993: "Why hunter-gatherers work: An ancient version of the problem of public goods [and comments and reply]". Current anthropology 34 (4): 341-361. https://doi.org/10.1086/204182

Henrikson, L. S. 2004: "Frozen bison and fur trapper's journals: building a prey choice model for Idaho's Snake River Plain”. Journal of Archaeological Science 31 (7): 903-916. https://doi.org/10.1016/j.jas.2003.12.005

Hesse, B. 1985: "Archaic exploitation of small mammals and birds in northern Chile". Estudios Atacameños 7: 42-61.

Hockett, B. S. et Bicho, N. F. 2000: "The rabbits of Picareiro Cave: small mammal hunting during the Late Upper Palaeolithic in the Portuguese Estremadura”. Journal of Archaeological Science 27 (8): 715 723. https://doi.org/10.1006/jasc.1999.049

Hockett, B. et Haws, J. A. 2002: "Taphonomic and methodological perspectives of leporid hunting during the Upper Paleolithic of the western Mediterranean Basin". Journal of Archaeological method and Theory 9 (3): 269-302.
Hockett, B. et Haws, J. 2003: "Nutritional ecology and diachronic trends in Paleolithic diet and health". Evolutionary Anthropology: Issues, News, and Reviews 12 (5): 211-216. https://doi.org/10.1002/evan.10116

Hockett, B. et Haws, J. A. 2005: "Nutritional ecology and the human demography of Neandertal extinction". Quaternary International 137 (1): 21-34. https://doi.org/10.1016/j.quaint.2004.11.017

Janssen, M. A. et Hill, K. 2014: "Benefits of grouping and cooperative hunting among ache hunter-gatherers: insights from an agent-based foraging model". Human ecology 42 (6): 823-835. https://doi.org/10.1007/s10745-014-9693-1

Jochim, M. 1987: "Late pleistocene refugia in Europe". In O. Schoffer (ed.): The Pleistocene Old World. Regional perspectives. Springer. Boston: 317-331.

Jones, E. L. 2006: "Prey choice, mass collecting, and the wild European rabbit (Oryctolagus cuniculus)". Journal of Anthropological Archaeology 25 (3): 275-289. https://doi.org/10.1016/j.jaa.2005.11.002

Jones, E. L. 2016: In search of the broad spectrum revolution in Paleolithic southwest Europe. Springer. New York. https://doi.org/10.1007/978-3-319-22351-3

Kelly, R. L. 2013: The lifeways of hunter-gatherers: the foraging spectrum. Cambridge University Press. Cambridge.

Kuhn, S. L. Stiner, M. C. 2006: "What's a mother to do? The division of labor among Neandertals and modern humans in Eurasia". Current Anthropology 47 (6): 953-980. https://doi.org/10.1086/507197

Lloveras, L.; Moreno-García, M. et Nadal, J. 2008a: "Taphonomic analysis of leporid remains obtained from modern Iberian lynx (Lynx pardinus) scats". Journal of Archaeological Science 35 (1): 1-13. https://doi.org/10.1016/j.jas.2007.02.005

Lloveras, L.; Moreno-Garcia, M. et Nadal, J. 2008b: “Taphonomic study of leporid remains accumulated by the Spanish Imperial Eagle (Aquila adalberti)". Geobios 41 (1): 91-100. https://doi.org/10.1016/j.geobios.2006.11.009

Lloveras, L.; Moreno-García, M. et Nadal, J. 2009: "The eagle owl (Bubo bubo) as a leporid remains accumulator: taphonomic analysis of modern rabbit remains recovered from nests of this predator". International Journal of Osteoarchaeology 19 (5): 573-592. https://doi.org/10.1002/oa.995

López-Martínez, N. 2008: "The lagomorph fossil record and the origin of the European rabbit”. In P. C. Alves, N. Ferrand et K. Hackländer (eds.): Lagomorph biology. Evolution, ecology, and conservation. Springer. Berlin, Heidelberg: 27-46.

Lupo, K. D. 2007: "Evolutionary foraging models in zooarchaeological analysis: recent applications and future challenges". Journal of archaeological research 15 (2): 143-189. https://doi.org/10.1007/s10814-007-9011-1

Lupo, K. D. et Schmitt, D. N. 2002: "Upper Paleolithic net-hunting, small prey exploitation, and women's work effort: a view from the ethnographic and ethnoarchaeological record of the Congo Basin". Journal of Archaeological Method and Theory 9 (2): 147-179.

Lupo, K. D. et Schmitt, D. N. 2016: "When bigger is not better: The economics of hunting megafauna and its implications for Plio-Pleistocene hunter-gatherers". Journal of Anthropological Archaeology 44: 185-197. https://doi.org/10.1016/j.jaa.2016.07.012

Lupo, K. D.; Schmitt, D. N. et Madsen, D. B. 2020: "Size matters only sometimes: the energy-risk trade-offs of Holocene prey acquisition in the Bonneville basin, western USA". Archaeological and Anthropological Sciences 12 (8): 1-18. https://doi.org/10.1007/s12520-020-01146-7

MacArthur, R. H. et Pianka, E. R. 1966: "On optimal use of a patchy environment". The American Naturalist 100 (916): 603-609.

Manne, T.; Cascalheira, J.; Évora, M.; Marreiros, J. et Bicho, N. 2012: "Intensive subsistence practices at Vale Boi, an Upper Paleolithic site in southwestern Portugal". Quaternary International 264: 83-99. https://doi.org/10.1016/j.quaint.2012.02.026

Marín Arroyo, A. B. 2008: "Demografía y cambio ambiental. Hipótesis para el cambio económico en el Cantábrico Oriental durante el Tardiglacial". Complutum 19 (1): 47-66.

Marín Arroyo, A. B. 2009: "Exploitation of the Montane zone of Cantabrian Spain during the Late Glacial: faunal evidence from El Mirón Cave". Journal of Anthropological Research 65 (1): 69-102. https://doi.org/10.3998/jar.0521004.0065.106 
Mix, A. C.; Bard, E. et Schneider, R. 2001: "Environmental processes of the ice age: land, oceans, glaciers (EPILOG)". Quaternary Science Reviews 20 (4): 627-657. https://doi.org/10.1016/S0277-3791(00)00145-1

Morin, E.; Bird, R. B. et Bird, D. 2020: "Mass procurement and prey rankings: insights from the European rabbit". Archaeological and Anthropological Sciences 12 (11): 1-14. https://doi.org/10.1007/s12520-020-01212-0

Morin, E.; Bird, D.; Winterhalder, B. et Bliege Bird, R. 2021: "Deconstructing Hunting Returns: Can We Reconstruct and Predict Payoffs from Pursuing Prey?" Journal of Archaeological Method and Theory (sous presse). https://doi.org/10.1007/s10816-021-09526-6

Morin, E.; Meier, J.; El Guennouni, K.; Moigne, A.M.; Lebreton, L.; Rusch, L.; ... et Cochard, D. 2019: "New evidence of broader diets for archaic Homo populations in the northwestern Mediterranean". Science Advances 5: 1-12. https://doi.org/10.1126/sciadv.aav9106

Parer, I. et Wood, D. H. 1986: "Further observations of the use of warren entrances as an index of the number of rabbits, Oryctolagus cuniculus". Australian Wildlife Research 13: 331-332. https://doi.org/10.1071/wr9860331

Pérez Ripoll, M. 2004: "La consommation humaine des lapins pendant le Paléolithique dans la région de Valencia (Espagne) et l'étude des niveaux gravétiens de La Cova de les Cendres (Alicante). In J.-P. Brugal et J. Desse (eds.): Petits animaux et sociétés humaines. Du complément alimentaire aux ressources utilitaire. Actes XXIV Rencontres Internationales d'Archéologie et d'Histoire d'Antibes 2004. Éd. APDCA. Antibes: 191-206.

Pérez Ripoll, M. 2005: “Caracterización de las fracturas antrópicas y sus tipologías en huesos de conejo procedentes de los niveles gravetienses de la Cova de les Cendres (Alicante)". Munibe AntropologiaArkeologia 57: 239-254

Pérez Ripoll, M. et Martínez Valle, R. 2001: "La caza, el aprovechamiento de las presas y el comportamiento de las comunidades cazadoras prehistóricas". In V. Villaverde (ed.): De neandertales a cromañones: el inicio del poblamiento en las tierras valencianas. Servei de Publicacions, Universitat de València. València: 73-98.

Real, C. 2020: "Rabbit: more than the Magdalenian main dish in the Iberian Mediterranean region. New data from Cova de les Cendres (Alicante, Spain)". Journal of Archaeological Science: Reports 32. https://doi.org/10.1016/j.jasrep.2020.102388

Riquelme, J. A.; Simon Vallejo, M. D. et Cortés Sánchez, M. 2005: "La fauna de mamíferos del Solutrense en la Cueva de Nerja". Munibe. Antropologia-arkeologia 57: 255-263.

Rufí, I.; Lloveras, L.; Soler, J. et Soler, N. 2020: "Small prey exploitation during the final Gravettian in the northeast of the Iberian Peninsula: The case of Level D from Arbreda Cave". International Journal of Osteoarchaeology 30 (3): 330-344. https://doi.org/10.1002/oa.2861

Simms, S. R. 1987: Behavioral ecology and hunter-gatherer foraging: an example from the Great Basin. British Archaeological Reports International Series 381. Oxford.

Sommer, R. S. et Nadachowski, A. 2006: "Glacial refugia of mammals in Europe: evidence from fossil records". Mammal Review 36 (4): 251 265. https://doi.org/10.1111/j.1365-2907.2006.00093.x

Speth, J. D. 2010: The Paleoanthropology and Archaeology of big-game hunting. Protein, fat, or Politics? Springer. New York. https://doi.org/10.1007/978-1-4419-6733-6

Stephens, D. W. et Krebs, J. R. 1986: Foraging theory. Princeton University Press. New Jersey.

Stiner, M. C. 2001: "Thirty years on the 'Broad Spectrum Revolution' and paleolithic demography". Proceedings of the National Academy of Sciences 98 (13): 6993-6996. https://doi.org/10.1073/pnas.121176198

Stiner, M. C.; Munro, N. D. et Surovell, T. A. 2000: "The tortoise and the hare - Small-game use, the broad-spectrum revolution, and paleolithic demography". Current Anthropology 41 (1): 39-73. https://doi.org/10.3732/ajb.92.1.142

Stiner, M. C. et Munro, N. D. 2002: "Approaches to prehistoric diet breadth, demography, and prey ranking systems in time and space". Journal of Archaeological Method and Theory 9 (2): 181-214. https://oi.org/10.1023/A:1016530308865
Stiner, M. C. et Kuhn, S. L. 2016: "Are we missing the "sweet spot" between optimality theory and niche construction theory in archaeology?". Journal of Anthropological Archaeology 44: 177-184. https://doi.org/10.1016/j.jaa.2016.07.006

Straus, L. G. 1987: "Upper Paleolithic ibex hunting in SW Europe". Journal of Archeological Science 14: 163-178. https://doi.org/10.1016/0305-4403(87)90004-5

Straus, L. G. 1992: Iberia before the Iberians: the stone age prehistory of Cantabrian Spain. University of New Mexico Press. Albuquerque.

Straus, L. G. 2013: "Iberian archaeofaunas and hominin subsistence during Marine Isotope Stages 4 and 3". In J. L. Clark et J. D. Speth (eds.) Zooarchaeology and modern human origins during Marine Isotope Stages 4 and 3. Springer. Dordrecht: 97-128. https://doi.org/10.1007/978-94-007-6766-9 7

Straus, L. G. 2018: "El Paleolítico Superior de la península ibérica”. Trabajos de Prehistoria 75 (1): 9-51. https://doi.org/10.3989/tp.2018.12202

Taberlet, P.; Fumagalli, L.; Wust-Saucy, A. G. et Cosson, J. F. 1998 : "Comparative phylogeography and postglacial colonization routes in Europe". Molecular ecology 7 (4): 453-464. https://doi.org/10.1046/j.1365-294x.1998.00289.x

Ugan, A. 2005: "Does size matter? Body size, mass collecting, and their implications for understanding prehistoric foraging behavior". American Antiquity 70, 1: 75-89. https://doi.org/10.2307/40035269

Verpoorte, A. 2009: "Limiting factors on early modern human dispersals: The human biogeography of late Pleniglacial Europe". Quaternary International 201 (1-2): 77-85. https://doi.org/10.1016/j.quaint.2008.05.021

Villafuerte, R. et Moreno, S. 1997: "Predation risk, cover type,and group size in European rabbits in Donana (SW Spain)". Acta Theriologica, 42 (2): 225-230. https://doi.org/10.4098/at.arch.97-23

Villaverde, V.; Martínez-Valle, R.; Guillem, P. M. et Fumanal, M. P. 1996: "Mobility and the role of small game in the Middle Paleolithic of the central region of the Spanish Mediterranean: a comparison of Cova Negra with other Paleolithic deposits". In E. Carbonell et M. Vaquero (eds.): The Last Neandertals/The first anatomically modern humans. Cultural change and human evolution: The crisis at $40 \mathrm{ka}$ $B P$. Universitat Rovira i Virgili. Igualada: 19-33.

Villaverde, V.; Román, D.; Martínez Valle, R.; Pérez Ripoll, M.; Badal, E.; Bergadà, M.; ...et Tormo, C. 2010: "El Paleolítico superior en el País Valenciano. Novedades y perspectivas". In X. Mangado (ed.): Jornadas Internacionales sobre el Paleolitico superior peninsular Novedades del siglo XXI (Barcelona 2010). Universitat de Barcelona. Barcelona: 85-113.

Villaverde, V.; Real, C.; Román, D.; Albert, R. M.; Badal, E.; Bel, M. Á. ...et Pérez-Ripoll, M. 2019: "The early Upper Palaeolithic of Cova de les Cendres (Alicante, Spain)". Quaternary International 515: 92124. https://doi.org/10.1016/j.quaint.2017.11.051

Winterhalder, B. 1981: "Foraging strategies in the boreal forest: an analysis of Cree hunting and gathering". In B. Winterhalder et E. A. Smith (eds.): Hunter-gatherer foraging strategies. Ethnographic and Archaeological Analyses. The University of Chicago Press. Chicago: 66-98.

Yravedra, J. 2006: "Implicaciones Tafonómicas y zooarqueológicas del consumo de lagomorfos por Búho Real (Bubo bubo) en la interpretación de los yacimientos arqueológicos". Anales de Prehistoria y Arqueología de la Universidad de Murcia 22: 33-47.

Yravedra, J.; Herranz D.; Sesé C.; López Cisneros P.; Linares-Matás G.; Pernas M.; ... et Álvarez-Alonso D. 2018: "Lagomorph exploitation during the Upper Palaeolithic in the northern Iberian peninsula. New evidence from Coímbre Cave (Asturias, Spain)". Quaternary International 506: 5-68. https://doi.org/10.1016/j.quaint.2018.06.016

Yravedra, J.; Andrés-Chaín, M.; Cacho, C.; Martos, J. A.; Marquer, L.; Avezuela, B.; ... et Valdivia, J. 2019: "Recurrent Magdalenian occupation in the interior of the Iberian Peninsula: new insights from the archaeological site of La Peña de Estebanvela (Segovia, Spain)". Archaeological and Anthropological Sciences 11 (4): 1477-1489. https://doi.org/10.1007/s12520-018-0620-z

Zeder, M. A. 2012: "The broad spectrum revolution at 40: resource diversity, intensification, and an alternative to optimal foraging explanations". Journal of Anthropological Archaeology 31 (3): 241-264. https://doi.org/10.1016/j.jaa.2012.03.003 